\title{
Periodontal disease of the mother and incidence of preeclampsia
}

\section{Thrasyvoulos-Marios Smyrlis, Sofoklis Stavros, Dimitrios Loutradis, Peter Drakakis}

$1^{\text {st }}$ OB/GYN Dept, Medical School, National and Kapodistrian University of Athens, Athens, Greece

Corresponding Author

Thrasyvoulos-Marios Smyrlis, DDS, Tel.: 6937686533, email: asmirlis@yahoo.gr

\section{Introduction}

Periodontitis is a chronic inflammatory disease, which is progressing in a slow pace and leads to gingival tissue destruction and alveolar bone resorption. If left untreated, it will lead gradually in tooth loss. It is caused by the simultaneous action of bacterial virulence factors and the response of the immune system $^{1,2}$. In the literature there is growing evidence of a possible link between periodontal disease and systemic health. The relationship between periodontitis and diabetes mellitus ${ }^{3}$, atherosclerosis and cardiovascular diseases ${ }^{4,5,6}$, renal disease ${ }^{7,8}$, cognitive impairment ${ }^{9,10}$, cancer ${ }^{11,12}$, metabolic syndrome ${ }^{13}$ and complications in pregnancy (premature birth ${ }^{14}$, low birth weight ${ }^{15,16}$ and preeclampsia) has been discussed in the literature. Preeclampsia is an important factor of mother and infant morbidity and mortality and it is affecting $5-7 \%$ of pregnancies ${ }^{17,18}$. Preeclampsia is defined as a rise in blood pressure (systolic pressure $\geq 140 \mathrm{mmHg}$ or diastolic pressure $\geq 90 \mathrm{mmHg}$ ) combined with proteinuria $(\geq 300 \mathrm{mg} / 24 \mathrm{~h})^{17,18}$. The cause of preeclampsia is unknown, however in the literature there are documented several risk factors such as antiphospholipid antibody syndrome, renal disease, prior preeclampsia, systemic lupus erythe- matosus, nulliparity, chronic hypertension, diabetes mellitus, high altitude, multiple gestations, strong family history of cardiovascular disease, obesity, family history of preeclampsia and advanced maternal age $^{18}$. It has been proposed that in the pathogenesis of preeclampsia, the immune system plays an important role. Given the role of inflammation in the pathogenesis of preeclampsia, it is suggested that the disease might have also an etiology of microbial origin ${ }^{19}$. In fact it has been proposed that the main dysregulations that accompany preeclampsia, which are abnormal trophoblast invasion of the placenta, anti-angiogenic responses, oxidative stress and inflammation, could be explained by an infection caused by microbes that are dormant and as a result difficult to detect ${ }^{18}$.

\section{Epidemiologic studies}

In the literature there is growing evidence of a link between periodontitis and preeclampsia. Until 2018, ten systematic reviews and meta-analyses have been conducted. Four reported a mild association and six a strong association between periodontitis and preeclampsia ${ }^{20}$. The majority of the scientific papers, which research the association between periodontitis 
and preeclampsia, are observational studies (89.6\%) and the intervention studies are the minority (10.4\%). However, although observational studies indicate an association between the aforementioned multifactorial diseases, intervention studies do not show reduction in the risk of preeclampsia after periodontal therapy during pregnancy ${ }^{21}$. This can be interpreted either as absence of etiologic link between periodontitis and preeclampsia or as if the pathologic mechanisms which link periodontitis and preeclampsia, preceded the time of the intervention or even pregnancy.

\section{Hypothetical pathogenic mechanism}

Two are the possible mechanisms, which explain the possible link between periodontitis and preeclampsia. Firstly periodontitis, which is characterized by chronic inflammation of the periodontium, the supportive structure of the tooth, results in incidents of bacteremia and finally dissemination of bacteria to the foetoplacental unit. There they cause an ectopic infection and/or trigger an inflammatory reaction which elevates levels of inflammatory cytokines and mediators, which ultimately result in preeclampsia ${ }^{22}$, probably by causing a shift in immune response, that causes failure of human trophoblast invasion and spiral artery remodeling. Secondly, cytokines and mediators produced by the inflamed periodontium enter the bloodstream through which they reach either the foetoplacental unit, where they enhance the accumulation of larger amounts of these mediators or the liver where they cause an inflammatory response with a production of CRP and fibrinogen ${ }^{22,23,24}$. The aforementioned cytokines and mediators eventually enter the foetoplacental unit and cause preeclampsia.

\section{Evidence in the literature supporting} the proposed mechanistic model

There is growing evidence in the literature, which support the proposed mechanistic model. Periodon- titis (but also gingivitis and daily activities such as chewing and tooth brushing, however in less extent) causes episodes of bacteremia ${ }^{25}$. Today we know that bacteria exist in anatomic areas, which were thought to be sterile. Broad-range PCR can detect prokaryotic rDNA in samples, which were negative in culture. Examples are endocarditis, interstitial cystitis, endophthalmitis, meningitis and orthopedic samples ${ }^{26}$. Placenta harbors its own unique microbiome. Placenta microbiome resembles the most the microbiome of the gingival plaque, tongue and tonsils. By contrast, the proximal anatomic sites and potential contaminants during delivery of the placenta (stool and vagina) bore no evidence of similarity. This may be evidence of a hematogenous establishment of the placental microbiome ${ }^{27}$. Four main classes of dysregulation characterize preeclampsia. Abnormal trophoblast invasion of the placenta, anti-angiogenic responses (increase of sFLT1 \& sEnd and decrease of PLGF), oxidative stress and inflammation (increase of IL-6, TNF $\alpha$ and decrease of IL-10), all of which may be caused by bacteria infection ${ }^{19}$. In in vitro studies periodontal pathogen microbe Porphyromonas gingivalis induces the apoptosis of human extravillous trophoblast derived HTR8 cells. Extravillous trophoblast cells play an important role in spiral artery remodeling ${ }^{28,29}$. Also periodontal pathogen Aggregatibacter actinomycetemcomitans LPS induce the apoptosis of human placental trophoblast cells ${ }^{30}$. In addition Porphyromonas gingivalis infection increased the placental Th1/Th2 cytokine ratio in mice ${ }^{31}$, decreased the numbers of decidua CD56 $6^{\text {bright }}$ $\mathrm{dNK}$ and $\mathrm{CD}^{2} 6^{+} \mathrm{dNK}$ cells ${ }^{32}$ and increased the numbers of Th17 lymphocytes and decreased the numbers of Treg lymphocytes in plasma of human patients with periodontitis ${ }^{33}$. These changes have been observed in preeclampsia ${ }^{31,32,32}$. Periodontal pathogenic bacteria Porphyromonas gingivalis, Fusobacterium nucleatum, Treponema denticola, Prevotella intermedia and Aggregatibacter (Actinobacillus) actinomycetem- 
comitans have been found in higher proportion in placenta of women with preeclampsia ${ }^{34}$. Also pregnant women with periodontitis have higher numbers of periodontal pathogenic bacteria in placenta ${ }^{35}$.

In patients with periodontitis serum levels of IL-1 $\beta$, IL-6, TNF- $\alpha$ and CRP are elevated ${ }^{36,37}$. These mediators are also elevated in preeclampsia ${ }^{38}$. It is proposed as mentioned above that these cytokines and mediators produced by the inflamed periodontium enter the bloodstream through which they reach either the foetoplacental unit, where they enhance the accumulation of larger amounts of these mediators or the liver where they cause an inflammatory response with a production of CRP and fibrinogen ${ }^{22,23,24}$. The aforementioned cytokines and mediators eventually enter the foetoplacental unit and cause preeclampsia by raising the topical levels of inflammatory cytokines and mediators and eventually cause an inflammatory reaction.

\section{Genetic factors linking periodontitis to preeclampsia}

Periodontitis and preeclampsia are both caused by a hyper inflammatory reaction of the immune system. The possible link between single nucleotide polymorphisms (SNPs) of IL1A, IL1B, IL1RN, IL6, IL10, vitamin
D receptor (VDR) and COX-2 genes and periodontitis or preeclampsia manifestation has been researched in the literature. Polymorphisms IL1A-889T, IL1B-3953/4T, IL6-174G, IL10-592C, IL10-819C, IL10-1082G, VDR ApaI A and VDR TAqI T increase the risk for periodontitis. IL1RN+2018C has no association or decreases the risk of periodontitis and for COX-2-765 literature presents conflicting results $39,40,41,42,43,44,45,46,47,48,49,50,51$. In the same time polymorphisms

IL1A-889C, IL10-819C and IL1RN+2018C increase the risk for preeclampsia, polymorphisms IL1B3953/4T and IL6-174G do not have any effect on the risk for preeclampsia, polymorphisms of COX-2 and VDR have not been studied yet and polymorphisms of IL10-592, IL10-1082 have had inconclusive results 52,53,54,55,56,57,58,59 (Table 1). In conclusion there could be a genetic linking factor between periodontitis and preeclampsia, especially regarding SNPs of IL10-592, IL10-819, IL10-1082 and COX-2 -765 but definitely more scientific data are needed in order to have solid evidence.

\section{Multifactorial nature of preeclampsia and possible microbial etiology}

Preeclampsia is believed to develop in two stages. In the first stage there is inadequate remodeling of

Table 1.

\begin{tabular}{|c|c|c|}
\hline & PERIODONTITIS & PREECLAMPSIA \\
\hline IL1A-889T(IL1A-889C) & $\uparrow(\downarrow)$ & $\downarrow(\uparrow)$ \\
\hline IL1B-3953/4T & 个 & $\mathbf{X}$ \\
\hline IL6-174G(IL6-174C) & $\uparrow^{a, c} / X^{c}$ & $\boldsymbol{\uparrow}^{\mathrm{e}} / \mathrm{X}$ \\
\hline IL10-592C(IL-10-592A) & $\uparrow(\downarrow)$ & $\boldsymbol{\uparrow}(\boldsymbol{\downarrow}) / \boldsymbol{\downarrow}(\boldsymbol{\uparrow})$ \\
\hline IL10-819C & $\uparrow$ & $\uparrow$ \\
\hline IL10-1082G(IL10-1082A) & $\boldsymbol{\uparrow}(\boldsymbol{\downarrow}) / \downarrow(\uparrow)$ & $\mathbf{X} / \downarrow(\boldsymbol{\uparrow})$ \\
\hline VDR ApaI A & $\uparrow$ & No evidence \\
\hline VDR TaqI T & $\uparrow$ & No evidence \\
\hline IL1RN+2018C & $\mathbf{X} / \downarrow$ & No evidence \\
\hline IL1RN rs315952C & $\mathbf{x}$ & $\uparrow$ \\
\hline COX-2 -765C & $\mathrm{X} / \boldsymbol{\downarrow} / \uparrow$ & $\downarrow$ \\
\hline
\end{tabular}


the spiral arteries in early gestation, which results in poor placental development. In the second stage, poor placental development results in ischemia/ reperfusion injury and oxidative stress, maternal endothelial cell dysfunction and finally to the clinical manifestations of preeclampsia. However, poor placentation can be present without preeclampsia and preeclampsia can occur in pregnancies with neonates large for dates. It is proposed, that for the development of preeclampsia and instigation of stage two, maternal and placental factors modify the risk either by increasing maternal susceptibility or by increasing placental loading respectively. Maternal factors, which increase maternal susceptibility, are genetic susceptibility, obesity, advanced maternal age, maternal hypertension and pre-pregnancy endothelial damage. Placental factors, which increase placental loading, are increased placental volume, increased placental mass (e.g. twins) and severe uteroplacental insufficiency. In addition infection and/or inflammation can, according to Pennington et al, and Kell and Kenny, serve to lower the threshold for preeclampsia in cases of inadequate placentation.

As stated before, although the scientific research has not concluded yet regarding the etiology of preeclampsia, the dysregulations that accompany preeclampsia could be explained by microbial infection ${ }^{19}$. A systematic review of epidemiological studies in 2008 found that any bacterial or viral infection was associated with a two-fold higher risk of developing preeclampsia compared to women without infection ${ }^{60}$. A meta-analysis of 2008 found that urinary tract infection and periodontal disease during pregnancy were associated with an increased risk of preeclampsia ${ }^{61}$. Recent studies have associated infections with high-risk human papilloma virus, Chlamydia trachomatis, periodontitis, Chlamydia pneumoniae and cytomegalovirus IgG seropositivity with preeclampsia. On the other hand, some studies have not found an association of preeclampsia with infection with cytomegalovirus, Chlamydia pneumonia, Herpes simplex virus 2, or respiratory tract infection. HBsAg carriage has even been found to be associated with a reduced incidence of preeclampsia ${ }^{62}$.

Although no association has been found between Toxoplasma gondii and preeclampsia ${ }^{62}$, spiramycin, which is an antibiotic prescribed for treating Toxoplasma gondii infection, led to a decrease in preeclampsia incidence

$(0,5 \%$ incidence of preeclampsia in the group who were treated with spiramycin and 5, 2\% incidence of preeclampsia in the control group; odds ratio $=0.092$ 95\% confidence interval 0.021, 0.399; $P<$ $0.001)^{63}$.Interestingly, spiramycin is also effective against periodontal pathogens ${ }^{64}$.

\section{Pregnancy, inflammation of periodontal tissues, cytokine and hormonal changes during pregnancy}

Immune system suppression and high levels of progesterone favor certain microbe growth in periodontium. This results in the well-established pregnancy induced gingivitis in second trimester (13-24 weeks of gestation) and third trimester (25-36 weeks of gestation) ${ }^{65}$. Inflammation and sex hormones result in increased vascular permeability and alter the effectiveness of the epithelial barrier to the oral microbiota ${ }^{29}$. This theoretically could enhance incidence of bacteremia. In mid third trimester $(\sim 32$ weeks of gestation) although progesterone levels remain high, immune system suppression weakens, because IL-10 and IFN- $\gamma$ levels rise again ${ }^{66}$. Interestingly, preeclampsia occurs after $20^{\text {th }}$ week of gestation and is more common after $32^{\text {th }}$ week of gestation ${ }^{67}$.

\section{Conclusion}

There is growing evidence about a possible link between periodontitis and preeclampsia. The evidence which support an association derive from 
observational studies, studies in animal models and in vitro studies. Experimental studies and substantial number of studies conducted in developed countries do not support such evidence. The cause of this discrepancy may lie in differences in periodontitis' definition, genetic factors, differences in sample size, and differences in studies' design. On the other hand, the cause may lie in the time microbes react with the host's immune system. Preeclampsia may be caused by periodontal bacteria, independently of the clinical parameters of periodontitis. Periodontal bacteria may also have colonized placenta and foetoplacental unit by bacteremia caused by other pathways than inflammation of periodontal pockets such as gingivitis and pericoronitis. This may explain the number of studies, which do not support an association in developed countries, in which there is optimal access to dental care. In addition, periodontitis can be divided in to two pathologic entities, chronic periodontics and aggressive periodontitis, in which the genetic background differs. Also preeclampsia is divided in early on-set preeclampsia and late on-set preeclampsia. In most of the studies these parameters were not taken into account. In addition in most of the studies third molars are not taken into account in the periodontitis assessment, however the third molar region might host deep pockets, which act a reservoir of anaerobic microbes. In conclusion, more studies with different design are needed in order to cast light in the relationship of periodontitis, periodontal bacteria and preeclampsia.

\section{References}

1. Mantzavinos Z, Vrotsos I. Clinical periodontology. Medical publications Litsas; 2002.

2. Tsuchida S, Satoh M, Takiwaki M, Nomura F. Ubiquitination in Periodontal Disease: A Review. Int. J. Mol. Sci. 2017, 18:1476-1491.

3. Taylor JJ, Preshaw PM, Lalla E. A review of the ev- idence for pathogenic mechanisms that may link periodontitis and diabetes. J Clin Periodontol 2013; 40 (Suppl. 14): S113-S134.

4. Bartova J, Sommerova P, Lyuya-Mi Y, et al. Periodontitis as a Risk Factor of Atherosclerosis. Journal of Immunology Research Volume 2014, Article ID 636893, 9 pages.

5. Amabile N, Susini G, Pettenati-Soubayroux I,et al. Severity of periodontal disease correlates to inflammatory systemic status and independently predicts the presence and angiographic extent of stable coronary artery disease. Journal of Internal Medicine 2008 263; 644-652.

6. Teeuw WJ, Slot DE, Susanto H, et al. Treatment of periodontitis improves the atherosclerotic profile: a systematic review and meta-analysis. J Clin Periodontol 2014; 41: 70-79.

7. Zhang J, Jiang H, Sun M, Chen J. Association between periodontal disease and mortality in people with CKD: a metaanalysis of cohort studies. BMC Nephrology 2017; 18:269-280.

8. Kshirsagar AV, Moss KL, Elter JR, Beck JD, Offenbacher S, Falk RJ. Periodontal disease is associated with renal insufficiency in the Atherosclerosis Risk In Communities (ARIC) study. Am J Kidney Dis. 2005 Apr; 45(4):650-7.

9. Tonsekar PP, Jiang SS, Yue G. Periodontal disease, tooth loss and dementia: Is there a link? A systematic review. Gerodontology. 2017 Jun;34(2):151-163.

10. Noble JM, Borrell LN, Papapanou PN, Elkind MS, Scarmeas N, Wright CB. Periodontitis is associated with cognitive impairment among older adults: analysis of NHANES-III. J Neurol Neurosurg Psychiatry. 2009 Nov;80(11):1206-11.

11. Javed $F$, Warnakulasuriya $S$. Is there a relationship between periodontal disease and oral cancer? A systematic review of currently available evidence. Crit Rev Oncol Hematol. 2016 Jan; 97:197-205.

12. Maisonneuve P, Amar S, Lowenfels AB. Peri- 
odontal disease, edentulism, and pancreatic cancer: a meta-analysis. Ann Oncol. 2017 May 1;28(5):985-995.

13. Watanabe K, Cho YD. Periodontal disease and metabolic syndrome: a qualitative critical review of their association. Arch Oral Biol. 2014 Aug;59(8):855-70.

14. Walia M, Saini N. Relationship between periodontal diseases and preterm birth: Recent epidemiological and biological data. Int J Appl Basic Med Res. 2015 Jan-Apr; 5(1): 2-6.

15. Marin C, Segura-Egea JJ, Martınez-Sahuquillo A, Bullon P. Correlation between infant birth weight and mother's periodontal status. J Clin Periodontol 2005; 32: 299-304.

16. Boggess KA, Beck JD, Murtha AP, Offenbacher S. Maternal periodontal disease in early pregnancy and risk for a small-for-gestational-age infant. Am J Obstet Gynecol. 2006 May;194(5):1316-22.

17. Ramma W, Ahmed A: Is inflammation the cause of preeclampsia? Biochem Soc Trans. 2011 Dec;39(6):1619-27.

18. Uzan J, Carbonnel M, Piconne O, Asmar R, Ayoubi J-M. Pre-eclampsia: pathophysiology, diagnosis, and management. Vascular Health and Risk Management. 2011;7:467-474.

19. Kell DB, Kenny LC. A Dormant Microbial Component in the Development of Preeclampsia. Front Med (Lausanne). 2016 Nov 29; 3:60.

20. Vivares-Builes AM, Rangel-Rincón LJ, Botero JE, Agudelo-Suárez AA. Gaps in Knowledge About the Association Between Maternal Periodontitis and Adverse Obstetric Outcomes: An Umbrella Review.J Evid Based Dent Pract. 2018 Mar;18(1):1-27.

21. Kunnen A, van Doormaal JJ, Abbas F, Aarnoudse JG, van Pampus MG, Faas MM. Periodontal disease and pre-eclampsia: a systematic review. J Clin Periodontol. 2010 Dec;37(12):1075-87.

22. Madianos PN, Bobetsis YA, Offenbacher S. Adverse pregnancy outcomes (APOs) and periodontal disease: pathogenic mechanisms. J Clin Periodontol 2013; 40 (Suppl. 14): S170-S180.

23. Herrera JA, Parra B, Herrera E, et al. Periodontal disease severity is related to high levels of $\mathrm{C}$-reactive protein in pre-eclampsia. Journal of Hypertension. 2007;25: 1459-1464.

24. Chandy S, Joseph K, Sankaranarayanan A,et al. Evaluation of C-Reactive Protein and Fibrinogen in Patients with Chronic and Aggressive Periodontitis: A Clinico-Biochemical Study. Journal of Clinical and Diagnostic Research : JCDR. 2017;11(3):ZC41-ZC45.

25. Forner L, Larsen T, Kilian M, Holmstrup P. Incidence of bacteremia after chewing, tooth brushing and scaling in individuals with periodontal inflammation. J Clin Periodontal 2006; 33: 401-407.

26. Kell D, Potgieter M, Pretorius E. Individuality, phenotypic differentiation, dormancy and 'persistence' in culturable bacterial systems: commonalities shared by environmental, laboratory, and clinical microbiology [version 2; referees:2approved,1 approved with reservations]. F1000Research 2015, 4:179.

27. Aagaard K, Ma J, Antony KM, Ganu R, Petrosino J, Versalovic J. The placenta harbors a unique microbiome.Sci Transl Med. 2014 May 21;6(237):237ra65.

28. Ren H, Li Y, Jiang H, Du M. Porphyromonas gingivalis induces IL-8 and IFN-gamma secretion and apoptosis in human extravillous trophoblast derived HTR8/SV neo cells via activation of ERK1/2 and p38 signaling pathways. Placenta $2016 ; 45: 8-15$.

29. Reyes L, Phillips P, Wolfe B, et al. Porphyromonas gingivalis and adverse pregnancy outcome. J Oral Microbiol. 2017 Sep 13;10(1):1374153.

30. Li Y, Shibata Y, Zhang L, Kuboyama N, Abiko Y. Periodontal pathogen Aggregatibacter actinomycetemcomitans LPS induces mitochondria-depen- 
dent-apoptosis in human placental trophoblasts. Placenta 2011; 32:11-19.

31. Lin D, Smith MA, Elter J, et al. Porphyromonas gingivalis Infection in Pregnant Mice Is Associated with Placental Dissemination, an Increase in the Placental Th1/Th2 Cytokine Ratio, and Fetal Growth Restriction. Infection and Immunity. 2003;71(9):5163-5168.

32. Fukui A, Yokota M, Funamizu A, et al. Changes of NK Cells in Preeclampsia. Am J Reprod Immunol 2012; 67: 278-286.

33. Reyes L, Phillips P, Wolfe B, et al. Porphyromonas gingivalis and adverse pregnancy outcome. J Oral Microbiol. 2017 Sep 13;10(1):1374153.

34. Swati P, Thomas B, Vahab SA, Kapaettu S, Kushtagi P. Simultaneous detection of periodontal pathogens in subgingival plaque and placenta of women with hypertension in pregnancy. Arch Gynecol Obstet. 2012 Mar;285(3):613-9.

35. Blanc V, O’Valle F, Pozo E, Puertas A, León R, Mesa F. Oral bacteria in placental tissues: increased molecular detection in pregnant periodontitis patients.Oral Dis. 2015 Oct;21(7):905-12.

36. Moutsopoulos N, Madianos P. Low-Grade Inflammation in Chronic Infectious Diseases Paradigm of Periodontal Infections. Ann. N.Y. Acad. Sci. 2006;1088: 251-264.

37. Da Silva HEC, Stefani CM, de Santos Melo N, et al. Effect of intra-pregnancy nonsurgical periodontal therapy on inflammatory biomarkers and adverse pregnancy outcomes: a systematic review with meta-analysis. Systematic Reviews 2017;6:197-209.

38. Taylor BD, Ness RB, Klebanoff MA et al. First and second trimester immune biomarkers in preeclamptic and normotensive women. Pregnancy Hypertens. 2016 Oct;6(4):388-393.

39. Nikolopoulos GK, Dimou NL, Hamodrakas SJ, Bagos PG. Cytokine gene polymorphisms in periodontal disease: a meta-analysis of 53 studies including 4178 cases and 4590 controls. J Clin Periodontol. 2008;35(9):754-67.

40. Deng JS, Qin P, Li XX, Du YH. Association between interleukin-1ß C (3953/4)T polymorphism and chronic periodontitis: evidence from a meta-analysis. Hum Immunol. 2013 Mar;74(3):371-8.

41. Ma L, Chu WM, Zhu J, Wu YN, Wang ZL. Interleukin$1 \beta(3953 / 4) \mathrm{C} \rightarrow \mathrm{T}$ polymorphism increases the risk of chronic periodontitis in Asians: evidence from a meta-analysis of 20 case-control studies. Arch Med Sci. 2015 Apr 25; 11(2):267-73.

42. Komatsu Y, Galicia JC, Kobayashi T, Yamazaki K, Yoshie H: Association of interleukin-1 receptor antagonist +2018 gene polymorphism with Japanese chronic periodontitis patients using a novel genotyping method. Int J Immunogenet. 2008 Apr;35(2):165-70.

43. Nibali L, Pelekos G, D'Aiuto F,et al.Influence of IL-6 haplotypes on clinical and inflammatory response in aggressive periodontitis. Clin Oral Investig. 2013 May;17(4):1235-42.

44. Song GG, Choi SJ, Ji JD, Lee YH. Association between tumor necrosis factor- $\alpha$ promoter $-308 \mathrm{~A} / \mathrm{G},-238$ $\mathrm{A} / \mathrm{G}$, interleukin- $6-174 \mathrm{G} / \mathrm{C}$ and $-572 \mathrm{G} / \mathrm{C}$ polymorphisms and periodontal disease: a meta-analysis. Mol Biol Rep. 2013 Aug;40(8):5191-203.

45. Gabriela Teixeira F, Mendonça SA, Menezes Oliveira K, et al. Interleukin-6 c.-174G\&gt;C Polymorphism and Periodontitis in a Brazilian Population. Mol Biol Int. 2014;2014:490308.

46. Albuquerque CM, Cortinhas AJ, Morinha FJ, Leitão JC, Viegas CA, Bastos EM. Association of the IL-10 polymorphisms and periodontitis: a meta-analysis Mol Biol Rep. 2012 Oct; 39(10):9319-29.

47. Zhong $Q$, Ding $C$, Wang $M$, Sun $Y, X u$ Y. Interleukin-10 gene polymorphisms and chronic/aggressive periodontitis susceptibility: a meta-analysis based on 14 case-control studies. Cytokine. 2012 Oct; 60(1):47-54.

48. Cullinan MP, Westerman B, Hamlet SM, et al. Pro- 
gression of periodontal disease and interleukin-10 gene polymorphism. J Periodontal Res. 2008 Jun; 43(3):328-33.

49. Ho YP, Lin YC, Yang YH, Ho KY, Wu YM, Tsai CC.Cyclooxygenase-2 Gene-765 single nucleotide polymorphism as a protective factor against periodontitis in Taiwanese. J Clin Periodontol. 2008 Jan;35(1):1-8.

50. Jiang L, Weng $\mathrm{H}$, Chen MY, Zhang $\mathrm{C}$, Zeng $\mathrm{XT}$.Association between cyclooxygenase-2 gene polymorphisms and risk of periodontitis: a meta-analysis involving 5653 individuals. Mol Biol Rep. 2014 Jul;41(7):4795-801.

51. Deng H, Liu F, Pan Y, Jin X, Wang H, Cao J. BsmI, TaqI, ApaI, and FokI polymorphisms in the vitamin D receptor gene and periodontitis: a meta-analysis of 15 studies including 1338 cases and 1302 controls. J Clin Periodontol. 2011 Mar;38(3):199-207.

52. Ghasemi Masoumeh, Kashani Elham, Fayyaz Azadeh, Attar Marzieh. Interleukin-1 alpha variation is associated with the risk of developing preeclampsia. European Journal of Obstetrics \& Gynecology and Reproductive Biology 2015;193:75-78.

53. Li J, Liu M, Zong J, et al. Genetic variations in IL1A and IL1RN are associated with the risk of preeclampsia in Chinese Han population. Scientific reports 2014; 4: 5250.

54. Ren R, Gao M, Fan P, Liu X, Liu R, Ma L, Chen Y, Liu Y, Bai H. Association study between -765G $>\mathrm{C}$ and $-1195 \mathrm{G}>$. A functional polymorphisms in the cyclooxygenase 2 gene and risk of preeclampsia.Zhonghua Yi Xue Yi Chuan Xue Za Zhi. 2015 Apr;32(2):245-9. doi: 10.3760/cma.j.i ssn.1003-9406.2015.02.021.

55. Gurdol F, Cakmakoglu B, Dasdemir S, Isbilen E, Bekpinar S, Isbir T. $-765 \mathrm{G} \rightarrow \mathrm{C}$ and $-1195 \mathrm{~A} \rightarrow \mathrm{G}$ promoter variants of the cyclooxygenase- 2 gene decrease the risk for preeclampsia. Genet Test Mol
Biomarkers. 2012 May;16(5):435-8.

56. Sowmya S, Ramaiah A, Nallari P, Jyothy A, Venkateshwari A. Role of IL-6 -174(G/C) promoter polymorphism in the etiology of early-onset preeclampsia. Inflamm Res. 2015 Jun;64(6):433-9.

57. Sowmya S,Manjari KS,Ramaiah A,et al. Interleukin 10 gene promoter polymorphisms in women with early-onset pre-eclampsia. Clin Exp Immunol 2014;178: 334-341.

58. Sowmya S, Ramaiah A, Nallari P, Jyothy A, Venkateshwari A. Role of IL-6 -174(G/C) promoter polymorphism in the etiology of early-onset preeclampsia. Inflamm Res. 2015 Jun;64(6):433-9.

59. Sowmya S, Ramaiah A, Sunitha T, Nallari P, Jyothy A, Venkateshwari A.Evaluation of Interleukin-10 (G-1082A) Promoter Polymorphism in Preeclampsia. J Reprod Infertil. 2013 Apr; 14(2):62-6.

60. Rustveld LO, Kelsey SF, Sharma R. Association between maternal infections and preeclampsia: a systematic review of epidemiologic studies. Matern Child Health J 2008, 12:223-242.

61. Conde-Agudelo A, Villar J, Lindheimer M. Maternal infection and risk of preeclampsia: systematic review and metaanalysis. Am J Obstet Gynecol 2008, 198:7-22.

62. Alvarado-Esquivel C,Vazquez-Alaniz F, Sandoval-Carrillo AA,et al. Lack of association between Toxoplasma gondii infection and hypertensive disorders in pregnancy: a case-control study in a Northern Mexican population. Parasites \& Vectors 2014, 7:167-173.

63. Todros T, Verdiglione P,Ogge G, Paladini D, Vergani P, Cardaropoli S. Low incidence of hypertensive disorders of pregnancy in women treated with spiramycin for toxoplasma infection. $\mathrm{Br} \mathrm{J}$ Clin Pharmacol 2005; 61(3):336-340.

64. Chiappe V, Gómez M, Fernández-Canigia L, Romanelli $\mathrm{H}$. The effect of spiramycin on Porphyromonas gingivalis and other "classic" periopathogens. Acta Odontol Latinoam. 2011;24(1):115-21. 
65. Reyes L, Phillips P, Wolfe B, et al. Porphyromonas gingivalis and adverse pregnancy outcome. J Oral Microbiol. 2017 Sep 13;10(1):1374153.

66. Shah NM, Herasimtschuk AA, Boasso A, et al. Changes in T Cell and Dendritic Cell Phenotype from Mid to Late Pregnancy Are Indicative of a Shift from Immune Tolerance to Immune Activation. Front. Immunol. 2017; 8:1138.
67. Arulkumaran N, Lightstone L. Severe pre-eclampsia and hypertensive crises. Best Pract Res Clin Obstet Gynaecol. 2013 Dec;27(6):877-84.

Received 25-7-2019

Revised 2-9-2019

Accepted 10-9-2019 